\title{
Additive prime number theory in an algebraic number field.
}

\author{
By Tikao TATUZAWA
}

(Received Nov. 15, 1955)

Thanks to the remarkable work of Vinogradov [7], we know that every sufficiently large odd integer can be expressed as a sum of three primes. Less attention has been paid to the problem of representing numbers in an algebraic number field as a sum of primes. Rademacher [4] carried over the Hardy-Littlewood formula in the rational case to a real quadratic number field on a certain hypothesis concerning the distribution of the zeros of Hecke's $\zeta(s, \lambda)$ funcfions.

Let $K$ be an algebraic number field of degree $n$ with $r_{1}$ real conjugates $K^{(l)}\left(l=1,2, \cdots, r_{1}\right)$ and $r_{2}$ pairs of conjugate complex conjugates $K^{(m)}, K^{\left(m+r_{2}\right)}\left(m=r_{1}+1, r_{1}+2, \cdots, r_{1}+r_{2}\right)$ so that $r_{1}+2 r_{2}=n$. Let $a, b$ be positive and $\mu, \nu$ be in $K$. For convenience, we use the symbol

$$
a\|\mu\| \leqq b\|\nu\|
$$

in the sense that

$$
a\left|\mu^{(i)}\right| \leqq b\left|\nu^{(i)}\right| \quad(i=1,2, \cdots, n) .
$$

For example, $\|\mu\| \leqq b$ means $\left|\mu^{(i)}\right| \leqq b$. Let $a$ be any principal ideal in $K$. By the theory of units, there exist a positive constant $c_{0}$ depending only on $K$ and at least one $\nu$ in $K$ such that

$$
\mathfrak{a}=(\nu) \text { and }\|\nu\| \leqq c_{0}{ }^{n} / N(\nu) \text {. }
$$

In what follows we fix this constant $c_{0}$. We use a letter $c$ to denote a positive constant depending only on $K$, not necessarily the same each time it occurs. The symbol

$$
Y=O(X)
$$


for positive $X$ means that there exists $c$ satisfying

$$
|Y| \leqq c X
$$

in the full domain under consideration. For example, the number of $\nu$ in (1) generating the same principal ideal $\mathfrak{a}$ is $O(1)$.

Let $\Gamma$ and $I$ be the integral domains consisting of all rational integers and all algebraic integers in $K$ respectively. An algebraic integer in the field is called a prime when the principal ideal generated by it is a prime ideal. In [6] Siegel considered the generalized Waring problem in an algebraic number field. He constructed the ring $J_{m}$ generated by $m$-th powers of all integers in the field. Using the generalized circle method, he proved that all totally positive integers in $J_{m}$ with sufficiently large norms are sums of $\left(2^{m-1}+n\right) m n+1$ integral $m$-th powers of totally positive numbers. Moreover he noticed that $J_{m}$ is an order in $I$, but not always equal to $I$, showing some theorems and some examples. Modeled on his idea, we construct the $\Gamma$-module $J$ generated by all primes in $K$. By means of Brun's sieve method, Hecke's prime ideal theorem, and Schnirelmann's density theorem, we will prove without any hypothesis that $J$ is of finite index in the additive group $I$ and every element in $J$ can be expressed as at most $c$ sums of primes.

\section{$\S 1$. On the number of representing integers in $K$ as a sum of two primes.}

Let $\mathfrak{b}_{1}, \mathfrak{b}_{2}, \cdots, \mathfrak{b}_{h}$ be representatives from ideal classes of $K$. Then any ideal $a$ in $K$ can be expressed in the form

$$
\mathfrak{a}=(\nu) \mathfrak{b}_{l}
$$

for some $\nu \in K$ with (1) and $\mathfrak{b}_{l}$. Let $\beta_{l 1}, \beta_{l 2}, \cdots, \beta_{l n}$ be an ideal basis of $\mathfrak{b}_{l}(l=1,2, \cdots, h)$. If we put $\alpha_{j}=\nu \beta_{l j}(j=1,2, \cdots, n)$, then

$$
\mathfrak{a}=\left(\alpha_{1}, \alpha_{2}, \cdots, \alpha_{n}\right)
$$

and

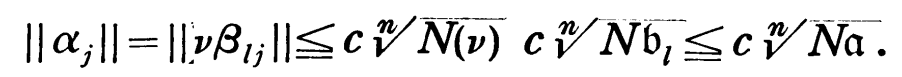


Let $\zeta$ be in $I$ with

$$
\|\zeta\| \leqq c_{1} \sqrt[n]{N(\zeta)}
$$

where

$$
c_{1}=6 c_{0}^{n}
$$

Let $\mathfrak{a}=\left(\alpha_{1}, \alpha_{2}, \cdots, \alpha_{n}\right)$ be an integral ideal in $K$ and $\beta \in I$. If we denote by $P(\mathfrak{a}, \zeta)$ the number of integers $\xi$ in $K$ subject to the conditions

$$
\xi \equiv \beta(\bmod . a), \quad\|\xi\| \leqq c_{1}\|\zeta\|, \quad\|\zeta-\xi\| \leqq c_{1}\|\zeta\|,
$$

then $P(\mathfrak{a}, \zeta)$ is the number of lattice points $\left(x_{1}, x_{2}, \cdots, x_{n}\right)$ in the $n$ dimensional euclidean space $S$ which lie in the domain

$$
\begin{aligned}
& \left|x_{1} \alpha_{1}^{(i)}+x_{2} \alpha_{2}^{(i)}+\cdots+x_{n} \alpha_{n}^{(i)}+\beta^{(i)}\right| \leqq c_{1}\left|\zeta^{(i)}\right| \\
& \left|x_{1} \alpha_{1}^{(i)}+x_{2} \alpha_{2}^{(i)}+\cdots+x_{n} \alpha_{n}^{(i)}+\beta^{(i)}-\zeta^{(i)}\right| \leqq c_{1}\left|\zeta^{(i)}\right|
\end{aligned}
$$

We change the variables as follows

$$
\begin{aligned}
u_{l} & =x_{1} \alpha_{1}^{(l)}+x_{2} \alpha_{2}^{(l)}+\cdots+x_{n} \alpha_{n}^{(l)}+\beta^{(l)}, \\
u_{m} & =x_{1} \Re\left(\alpha_{1}^{(m)}\right)+x_{2} \Re\left(\alpha_{2}^{(m)}\right)+\cdots+x_{n} \Re\left(\alpha_{n}^{(m)}\right)+\Re\left(\beta^{(m)}\right), \\
u_{m+r_{2}} & =x_{1} \mathfrak{\Im}\left(\alpha_{1}^{\left(m+r_{2}\right)}\right)+x_{2} \mathfrak{\Im}\left(\alpha_{2}^{\left(m+r_{2}\right)}\right)+\cdots+x_{n} \Im\left(\alpha_{n}^{\left(m+r_{2}\right)}\right)+\Im\left(\beta^{\left(m+r_{2}\right)}\right) .
\end{aligned}
$$

The domain (5) is now described by

$$
\begin{gathered}
\left|u_{l}\right| \leqq c_{1}\left|\zeta^{(l)}\right|, \quad\left|u_{l}-\zeta^{(l)}\right| \leqq c_{1}\left|\zeta^{(l)}\right| \\
u_{m}^{2}+u_{m+r_{2}}^{2} \leqq c_{1}^{2}\left|\zeta^{(m)}\right|^{2}, \quad\left(u_{m}-\mathfrak{R}\left(\zeta^{(m)}\right)\right)^{2}+\left(u_{m+r_{2}}-\Im\left(\zeta^{\left(m+r_{2}\right)}\right)\right)^{2} \leqq c_{1}^{2}\left|\zeta^{(m)}\right|^{2}
\end{gathered}
$$

Since

$$
\left|\frac{\partial\left(u_{1}, u_{2}, \cdots, u_{n}\right)}{\partial\left(x_{1}, x_{2}, \cdots, x_{n}\right)}\right|=\frac{\sqrt{d} N a}{2^{r_{2}}}
$$

where $d$ is the discriminant of $K$, taking a basis of a as in (2), we obtain 


$$
\begin{aligned}
& \iint_{u_{l} \in L_{l},} \cdots \cdots \int_{\left(u_{m}, u_{m+r_{\mathbf{2}}}\right) \in C_{m}} \frac{2^{r_{2}}}{\sqrt{ } d N \mathfrak{a}} d u_{1} d u_{2} \cdots d u_{n}<P(\mathfrak{a}, \zeta) \\
& <\iint_{u_{l} \in L_{l,}^{\prime}\left(u_{m}, u_{m+r_{2}}\right) \in C_{m}^{\prime}} \cdots \frac{2^{r_{2}}}{\sqrt{ } d N a} d u_{1} d u_{2} \cdots d u_{n},
\end{aligned}
$$

where $L_{l}, L_{l}^{\prime}$ are line segments with lengths

$$
\left(2 c_{1}-1\right)\left|\zeta^{(l)}\right|+O\left(v^{n} N a\right)
$$

and $C_{m}, C_{m}^{\prime}$ are common domains surrounded by two circles having radii $c_{1}\left|\zeta^{(m)}\right|+O(\sqrt[n]{N a})$ and central distances $\left|\zeta^{(m)}\right|$ so that with areas

$$
\left(2 c_{1}^{2} \sin ^{-1} \sqrt{1-\frac{1}{4 c_{1}^{2}}}-\sqrt{c_{1}^{2}-\frac{1}{4}}\right)\left|\zeta^{(m)}\right|^{2}+O\left(\left|\zeta^{(m)}\right| V^{n} N a+(\sqrt[n]{N a})^{2}\right) .
$$

From (3), we can easily deduce

$$
P(\mathfrak{a}, \zeta)=c \frac{N(\zeta)}{N a}+O\left(\left(\begin{array}{c}
N(\zeta) \\
N a
\end{array}\right)^{1-\frac{1}{n}}+1\right)
$$

This is a slight extention of Rademacher's work [5].

Let $\mathfrak{p}_{1}, \mathfrak{p}_{2}, \cdots, \mathfrak{p}_{k}$ be prime ideals in $K$. We denote by $P\left(\mathfrak{a}, \zeta ; \mathfrak{p}_{1}\right.$, $\mathfrak{p}_{2}, \cdots, \mathfrak{p}_{k}$ ) the number of integers in $K$ satisfying

$$
\begin{gathered}
\xi \equiv \beta(\bmod \cdot \mathfrak{a}), \quad\|\xi\| \leqq c_{1}\|\zeta\|, \quad\|\zeta-\xi\| \leqq c_{1}\|\zeta\| \\
\xi \notin \mathfrak{p}_{s}, \quad \zeta-\xi \notin \mathfrak{p}_{s} \quad(s=1,2, \cdots, k) .
\end{gathered}
$$

If we define $v_{s}=2$ if $\zeta \notin \mathfrak{p}_{s}$ and $v_{s}=1$ if $\zeta \in \mathfrak{p}_{s}$, then

$$
P\left(\mathfrak{a}, \zeta ; \mathfrak{p}_{1}, \mathfrak{p}_{2}, \cdots, \mathfrak{p}_{k}\right)=P\left(\mathfrak{a}, \zeta ; \mathfrak{p}_{1}, \mathfrak{p}_{2}, \cdots, \mathfrak{p}_{k-1}\right)-v_{k} P\left(\mathfrak{a} \mathfrak{p}_{k}, \zeta ; \mathfrak{p}_{1}, \mathfrak{p}_{2}, \cdots, \mathfrak{p}_{k-1}\right)
$$

By iteration,

$$
\begin{aligned}
& P\left(\mathfrak{a}, \zeta ; \mathfrak{p}_{1}, \mathfrak{p}_{2}, \cdots, \mathfrak{p}_{k}\right)=P(\mathfrak{a}, \zeta)-\sum_{1 \leqq s_{1} \leqq k} v_{s_{1}} P\left(\mathfrak{a} \mathfrak{p}_{s_{1}}, \zeta ; \mathfrak{p}_{1}, \mathfrak{p}_{2}, \cdots, \mathfrak{p}_{s_{1}-1}\right) \\
& \quad=P(\mathfrak{a}, \zeta)-\sum_{1 \leqq s_{1} \leqq k} v_{s_{1}} P\left(\mathfrak{a} \mathfrak{p}_{s_{1}}, \zeta\right)+\sum_{1 \leqq s_{2}<s_{1} \leqq k} v_{s_{1}} v_{s_{2}} P\left(\mathfrak{a p _ { s _ { 1 } }} \mathfrak{p}_{s_{2}}, \zeta ; \mathfrak{p}_{1}, \mathfrak{p}_{2}, \cdots, \mathfrak{p}_{s_{2}-1}\right) .
\end{aligned}
$$

Consequently, making use of Brun's method and taking $\mathfrak{a}=I$, we get 


$$
P\left(I, \zeta ; \mathfrak{p}_{1}, \mathfrak{p}_{2}, \cdots, \mathfrak{p}_{k}\right) \leqq P(I, \zeta)+\sum_{q=1}^{2 t}(-1)^{q} \sum_{s_{1}, s_{2}, \cdots, s_{q}} v_{s_{1}} v_{s_{2}} \cdots v_{s_{q}} P\left(\mathfrak{p}_{s_{1}} \mathfrak{p}_{s_{2}} \cdots \mathfrak{p}_{s_{q}}, \zeta\right)
$$

where $s_{q}(q=1,2, \cdots, 2 t)$ runs over

$$
\begin{gathered}
0<s_{2 t}<s_{2 t-1}<\cdots<s_{2}<s_{1}, \\
s_{q} \leqq k_{[(q-1) / 2]}
\end{gathered}
$$

for suitably chosen

$$
0=k_{t}<k_{t-1}<\cdots<k_{1}<k_{0}=k .
$$

By the aid of (6), therefore, we obtain

$$
\begin{aligned}
P\left(I, \zeta ; \mathfrak{p}_{1}, \mathfrak{p}_{2}, \cdots, \mathfrak{p}_{k}\right) & =O\left(N(\zeta) \sum_{q=0}^{2 t}(-1)^{q} \sum_{s_{1}, s_{2}, \cdots, s_{q}} \gamma_{s_{1}} \gamma_{s_{2}} \cdots \gamma_{s_{q}}\right) \\
& +O\left(N(\zeta)^{1-\frac{1}{n}} \prod_{r=0}^{t-1}\left(2 k_{r}\right)^{2}\right)
\end{aligned}
$$

with the abbreviation $\gamma_{s}=v_{\mathrm{s}} / N \mathfrak{p}_{s}$.

Now we assume

$$
11 \leqq N \mathfrak{p}_{1} \leqq N \mathfrak{p}_{2} \leqq \cdots \leqq N \mathfrak{p}_{k}
$$

Obviously

$$
\frac{4}{5}<\frac{9}{11} \leqq 1-\gamma_{s}
$$

First we set $k_{0}=k$. Next we choose $k_{r}$ in (7) such that

$$
\frac{4}{5} \leqq \prod_{k_{r}} \prod_{\left\langle s \leqq k_{r-1}\right.}\left(1-\gamma_{s}\right) \quad(r=1,2, \cdots, t),
$$

$$
\prod_{k_{r} \leqq s \leqq k_{r-1}}\left(1-\gamma_{s}\right)<\frac{4}{5} \quad(r=1,2, \cdots, t-1) .
$$

Denoting by $T_{r}$ the right hand side of the first inequality in (10) and using Theorem 79 of [3], we obtain

$$
\left|\sum_{q=0}^{2 t}(-1)^{q} \sum_{\substack{s_{q}<\cdots<s_{2}<s_{1} \\ s_{q} \leqq k_{[(q-1) / 2]}}} \gamma_{s_{1}} \gamma_{s_{2}} \cdots \gamma_{s_{q}}\right|<2 \prod_{r=1}^{t} T_{r} .
$$


Inserting this in (8), we get

$$
P\left(I, \zeta ; \mathfrak{p}_{1}, \mathfrak{p}_{2}, \cdots, \mathfrak{p}_{k}\right)=O\left(N(\zeta) \prod_{r=1}^{t} T_{r}\right)+O\left(N(\zeta)^{1-\frac{1}{n}} \prod_{r=0}^{t-1}\left(2 k_{r}\right)\right)
$$

For brevity we write

$$
Q=\prod_{s=1}^{k}\left(1-\frac{1}{N \mathfrak{p}_{s}}\right)
$$

On account of (9) and (10),

$$
\begin{aligned}
& \left(\prod_{s=1}^{k_{r}}\left(1-\frac{1}{N \mathfrak{p}_{s}}\right)\right)^{-1}=\frac{1}{Q} \prod_{j=1}^{r} \prod_{k_{j}<s \leqq k_{j-1}}\left(1-\frac{1}{N \mathfrak{p}_{s}}\right) \leqq \frac{1}{Q} \sqrt[3]{\prod_{j=1}^{r} T_{j}} \\
& <\frac{1}{Q} \sqrt[3]{\prod_{j=1}^{r} \frac{4}{5}\left(1-\gamma_{j}\right)^{-1}}<\frac{1}{Q}\left(1-\frac{1}{135}\right)^{r}(r=0,1, \cdots, t-1) .
\end{aligned}
$$

Let $\pi(x)$ be the number of all prime ideals having norms not exceeding $x$. It is well known that

$$
\pi(x)<c \frac{x}{\log x}
$$

and

$$
\frac{c}{\log x}<\sum_{N \leqq x}\left(1-\frac{1}{N \mathfrak{p}}\right)<\frac{c}{\log x}
$$

Now we take all prime ideals $\mathfrak{p}$ satisfying $11 \leqq N \mathfrak{p} \leqq{ }^{c_{2}} \sqrt{N(\zeta)}$ as $\mathfrak{p}_{1}$, $\mathfrak{p}_{2}, \cdots, \mathfrak{p}_{k}$, where $c_{2}$ is decided later. By (13),

$$
s \leqq \pi\left(N \mathfrak{p}_{s}\right)<c \frac{N \mathfrak{p}_{s}}{\log N \mathfrak{p}_{s}}<c N \mathfrak{p}_{s}
$$

From (12) and (15), with the help of the second inequality of (14), we have

$$
\log 2 k_{r}<\frac{c}{Q}\left(1-\frac{1}{135}\right)^{r}
$$

If follows from this that 


$$
\prod_{r=0}^{t-1}\left(2 k_{r}\right)^{2}<\exp \left(\frac{c}{Q}\right),
$$

whereas, employing the first inequality, we have

$$
\frac{1}{Q}=\left(\prod_{11 \leqq N \mathfrak{1} \leqq c_{2} \sqrt{ }(\zeta)}\left(1-\frac{1}{N \mathfrak{p}}\right)\right)^{-1}<c \log { }^{c_{2}} \sqrt{N(\zeta)}
$$

Hence, by suitably chosen $c_{2}$, we obtain

$$
\prod_{r=0}^{t-1}\left(2 k_{r}\right)^{2}<N(\zeta)^{\frac{1}{2 n}}
$$

On the other hand,

$$
\begin{aligned}
\prod_{r=1}^{t} T_{r} & =\prod_{s=1}^{k}\left(1-\frac{v_{s}}{N \mathfrak{p}_{s}}\right) \leqq \prod_{s=1}^{k}\left(1-\frac{1}{N \mathfrak{p}_{s}}\right)^{v_{s}} \\
& =\prod_{s=1}^{k}\left(1-\frac{1}{N \mathfrak{p}_{s}}\right)^{2} \prod_{\zeta \in \mathfrak{p}}\left(1-\frac{1}{N \mathfrak{p}_{s}}\right)^{-1} \leqq Q^{2} \prod_{\zeta \in \mathfrak{p}}\left(1-\frac{1}{N \mathfrak{p}}\right)^{-1} \\
& =Q^{2} \prod_{\zeta \in \mathfrak{b}}\left(1-\frac{1}{N \mathfrak{p}^{2}}\right)^{-1} \prod_{\zeta \in \mathfrak{p}}\left(1+\frac{1}{N \mathfrak{p}}\right)<c Q^{2} \prod_{\zeta \in \mathfrak{a}} \frac{1}{N \mathfrak{a}} \\
& <\frac{c}{\log ^{2} N(\zeta)} \sum_{\zeta \in \mathfrak{a}} \frac{1}{N \mathfrak{a}},
\end{aligned}
$$

by the second inequality of (14). Inserting (16) and (17) in (11), we get

$$
P\left(I, \zeta ; \mathfrak{p}_{1}, \cdots, \mathfrak{p}_{k}\right)=O\left(\frac{N(\zeta)}{\log ^{2} N(\zeta)} \sum_{\zeta \in \mathfrak{a}} \frac{1}{N \mathfrak{a}}\right)
$$

Let $P(\zeta)$ be the number of solutions of

$$
\zeta=\lambda+\mu, \quad\|\lambda\| \leqq c_{1}\|\zeta\|, \quad\|\mu\| \leqq c_{1}\|\zeta\|
$$

where $\lambda$ and $\mu$ are primes with

$$
\|\lambda\| \leqq c_{0} \sqrt[n]{N(\lambda)}, \quad\|\mu\| \leqq c_{0} \sqrt[n]{N(\bar{\lambda})} .
$$

If $N(\lambda)$ and $N(\mu)>{ }^{c_{2}} \sqrt{ } N(\zeta)$ in (19), then neither $\lambda$ nor $\mu$ is divided by any prime ideal satisfying $11 \leqq N \mathfrak{p} \leqq^{c_{2}} \sqrt{ } N(\zeta)$. By (18), the number of solutions in this case is 


$$
O\left(\begin{array}{ccc}
N(\zeta) & \cdot & 1 \\
\log ^{2} N(\zeta) & \sum_{\zeta \in \mathfrak{a}} & N \mathfrak{a}
\end{array}\right)
$$

On the other hand, in the case of $N(\lambda)$ or $N(\mu) \leqq{ }^{c_{2}} \sqrt{ } N(\zeta)$, the number of solutions is

$$
O\left(\pi\left({ }^{c_{2}} \sqrt{ } N(\zeta)\right)\right)=O\left(\frac{N(\zeta)}{\log ^{2} N(\zeta)}\right)
$$

in virtue of $\left[(20)\right.$ and $c_{2}>1$.

Collecting these results, we get

THEOREM 1. Let $\zeta$ be an algebraic integer in $K$ with

$$
\|\zeta\| \leqq c_{1} \stackrel{n}{\nu} N(\zeta)
$$

Let $P(\zeta)$ be the number of solutions of

$$
\zeta=\lambda+\mu,
$$

where $\lambda$ and $\mu$ are primes fulfilling the conditions

$$
\|\lambda\| \leqq c_{0} \sqrt[n]{ } N(\lambda), \quad c_{1}\|\zeta\| ; \quad\|\mu\| \leqq c_{0} \sqrt[n]{N(\mu)}, \quad c_{1}\|\zeta\| .
$$

Then

$$
P(\zeta)=O\left(\frac{N(\zeta)}{\log ^{2} N(\zeta)} \sum_{\zeta \in a} \begin{array}{c}
1 \\
N a
\end{array}\right)
$$

$\S 2$. On the density of the set consisting of integers in $K$ represented by a sum of two primes.

From Theorem 1, we obtain

$$
\begin{aligned}
& \sum_{N(\zeta) \leqq y} P^{2}(\zeta)=O\left(\sum_{N(\zeta) \leqq y} \frac{N(\zeta)^{2}}{\log ^{4} N(\zeta)} \sum_{\zeta \in \mathfrak{a}} \frac{1}{N a} \sum_{\zeta \in \mathfrak{b}} \begin{array}{c}
1 \\
N \mathfrak{b}
\end{array}\right) \\
& =O\left(\frac{y^{2}}{\log ^{4} y} \sum_{N(\zeta) \leqq y}\left(\sum_{\zeta \in a} \frac{1}{N \mathfrak{a}} \sum_{\zeta \in \mathfrak{b}} \begin{array}{c}
1 \\
N \mathfrak{b}
\end{array}\right)\right) \\
& =O\left(\frac{y^{2}}{\log ^{4} y} \sum_{\substack{N a \leq y \\
N b \leq y}} \frac{1}{N \mathfrak{a} N \mathfrak{b}} \sum_{\substack{N(\zeta) \leqq y \\
\zeta \in\{a, b\}}} 1\right),
\end{aligned}
$$


where $\{\mathfrak{a}, \mathfrak{b}\}$ is the least common multiple of $\mathfrak{a}$ and $\mathfrak{b}$, so that $\sqrt{ } N \mathfrak{a} N \bar{b} \leqq N\{\mathfrak{a}, \mathfrak{b}\}$. Since

$$
\sum_{\substack{N(\zeta) \leqq y \\ \zeta \in\{a, b\}}} 1=O\left(\frac{y}{N\{\mathfrak{a}, \mathfrak{b}\}}\right)=O\left(\frac{y}{\sqrt{N \mathfrak{a} N \mathfrak{b}}}\right),
$$

we obtain

$$
\sum_{N(\zeta) \leqq y} P^{2}(\zeta)=O\left(\frac{y^{3}}{\log ^{4} y}\right) \quad(2 \leqq y)
$$

Now we consider the sets

$$
L=\left\{\lambda:\left(\frac{1}{2 c_{0}}\right)^{n} y \leqq N(\lambda) \leqq\left(\frac{2}{3 c_{0}}\right)^{n} y, \quad\|\lambda\| \leqq c_{0} V^{n} N \overline{(\lambda)}, \lambda \text { prime }\right\}
$$

and

$$
M=\left\{\mu:\left(\frac{1}{6 c_{0}{ }^{n+1}}\right)^{n} y \leqq N(\mu) \leqq\left(\frac{1}{3 c_{0}{ }^{n+1}}\right)^{n} y,\|\mu\| \leqq c_{0} V^{n} N \overline{(\mu)}, \mu \text { prime }\right\}
$$

for $2 \cdot 6^{n} c_{0}^{n(n+1)} \leqq y$. If we put

$$
\zeta=\lambda+\mu
$$

then

$$
\begin{aligned}
\|\zeta\| & =\|\lambda+\mu\| \leqq c_{0}(\stackrel{n}{N(\lambda)}+\sqrt[n]{ } N(\mu)) \\
& \leqq c_{0}\left(\frac{2}{3 c_{0}}+\frac{1}{3 c_{0}{ }^{n+1}}\right) \sqrt[n]{y} \leqq \sqrt[n]{y}
\end{aligned}
$$

and

$$
N(\zeta) \leqq y
$$

It is easy to see

$$
\frac{1}{c_{0}^{n-1}} \sqrt[n]{ } N(\lambda) \leqq\|\lambda\|,
$$

whence follows 


$$
\frac{1}{6 c_{0}^{n}} \sqrt[n]{y}=-\frac{1}{c_{0}^{n-1}} \underset{2 c_{0}}{1} \sqrt[n]{y}-c_{0} \frac{1}{3 c_{0}^{n+1}} V^{n} y \leqq \frac{1}{c_{0}^{n-1}} \eta^{n} N\left(\overline{\lambda)}-c_{0} \sqrt[n]{N(\mu)}\right.
$$

$$
\leqq\|\lambda\|-\|\mu\| \leqq\|\zeta\|
$$

By (22) and (24),

$$
\left.\frac{1}{6 c_{0}^{n}}\|\zeta\| \leqq \frac{1}{6 c_{0}^{n}} \eta y \leqq N / \zeta\right)
$$

or

$$
\|\zeta\| \leqq c_{1} \sqrt[n]{\overline{N(\zeta)}}
$$

by (4). Because of (24),

$$
\frac{1}{4 c_{0}^{n}}\|\lambda\| \leqq \frac{1}{4 c_{0}^{n-1}} V N(\lambda) \leqq \frac{1}{4 c_{0}^{n-1}} \frac{2}{3 c_{0}} V^{n} y \leqq \frac{1}{6 c_{0}^{n}} \eta^{n} y\|\zeta\|
$$

whence follows

$$
\|\lambda\| \leqq c_{1}\|\zeta\|
$$

by (4). Moreover, by (24),

$$
\frac{1}{2}\|\mu\| \leqq \frac{c_{0}}{2} \sqrt[n]{ } N(\mu) \leqq \frac{c_{0}}{2} \frac{1}{3 c_{0}^{n+1}} \sqrt[n]{ } y=\frac{1}{6 c_{0}^{n}} \eta^{n} y \leqq\|\zeta\|
$$

so that

$$
\|\mu\| \leqq c_{1}\|\zeta\| \text {. }
$$

We see that the conditions in Theorem 1 are satisfied by (25), (26) and (27). In view of (23), we can deduce that

$$
\text { the number of }\{\lambda+\mu: \lambda \in L, \mu \in M\} \leqq \sum_{N(\zeta) \leqq y} P(\zeta) \text {. }
$$

Let $\pi(x, H)$ be the number of principal prime ideals whose norms do not exceed $x$. Then the left hand side of (28) is greater than

$$
c\left(\pi\left(c_{3} y, H\right)-\pi\left(c_{4} y, H\right)\right)\left(\pi\left(c_{5} y, H\right)-\pi\left(c_{6} y, H\right)\right)
$$

with abbreviations

$$
c_{3}=\left(\begin{array}{c}
2 \\
3 c_{0}^{-}
\end{array}\right)^{n}, \quad c_{4}=\left(\begin{array}{c}
1 \\
2 c_{0}
\end{array}\right)^{n}, \quad c_{5}=\left(\frac{1}{3 c_{0}^{n+1}}\right)^{n}, \quad c_{6}=\left(\frac{1}{6 c_{0}^{n+1}}\right)^{n} .
$$


If we use Hecke's prime ideal theorem [2], then

$$
O(1)=\sum_{\substack{c_{4} y \leqq N \mathfrak{p} \leqq c_{8} y \\ \mathfrak{p e} H}} \frac{\log N \mathfrak{p}}{N \mathfrak{p}} \leqq\left(\pi\left(c_{3} y, H\right)-\pi\left(c_{4} y, H\right)\right) \frac{\log c_{4} y}{c_{4} y}
$$

and

$$
O(1)=\sum_{\substack{c_{6} y \leqq N \mathfrak{N} \leqq c_{5} y \\ \mathfrak{p e H}}} \frac{\log N \mathfrak{p}}{N \mathfrak{p}} \leqq\left(\pi\left(c_{5} y, H\right)-\pi\left(c_{6} y, H\right)\right) \frac{\log c_{6} y}{c_{6} y}
$$

whence follows

$$
c \frac{y^{2}}{\log { }^{2} y}<\sum_{N(\zeta) \leqq y} P(\zeta) \quad\left(2 \cdot 6^{n} c_{0}^{n(n+1)} \leqq y\right)
$$

by (28).

Now we denote by $U(y)$ the number of

$$
\begin{aligned}
& \left\{\zeta: N(\zeta) \leqq y ; \zeta=\lambda+\mu ; \lambda, \mu \text { primes } ;\|\zeta\| \leqq c_{1} \sqrt{ } N(\zeta)\right. \\
& \left.\|\lambda\| \leqq c_{0} \sqrt{n} \overline{N(\lambda)}, \quad c_{1}\|\zeta\| ;\|\mu\| \leqq c_{0} \sqrt{N(\mu)}, \quad c_{1}\|\zeta\|\right\}
\end{aligned}
$$

Then, by (21) and (29),

$$
c \frac{y^{4}}{\log ^{4} y}<\left(\sum_{N(\zeta) \leqq y} P(\zeta)\right)^{2} \leqq U(y) \sum_{N(\zeta) \leqq y} P^{2}(\zeta)<U(y) c \frac{y^{3}}{\log ^{4} y} \cdot
$$

This gives

$$
c y<U(y)
$$

for sufficiently large $y$.

We write

$$
\begin{gathered}
A(x)=\text { the number of }\{\zeta:\|\zeta\| \leqq x ; \zeta \in I\} \\
E(x)=\text { the number of }\{\zeta:\|\zeta\| \leqq x ; \zeta=\lambda+\mu ; \lambda, \mu \text { primes; } \\
\left.\|\lambda\|,\|\mu\| \leqq c_{1} x\right\}
\end{gathered}
$$

Let $\omega_{1}, \omega_{2}, \cdots, \omega_{n}$ be an integral basis of $K$. Then $A(x)$ is the number of lattice points $\left(x_{1}, x_{2}, \cdots, x_{n}\right)$ in $S$ satisfying

$$
\left|x_{1} \omega_{1}^{(i)}+x_{2} \omega_{2}^{(i)}+\cdots+x_{n} \omega_{n}^{(i)}\right| \leqq x \quad(i=1,2, \cdots, n),
$$




$$
x_{j} \in \Gamma \quad(j=1,2, \cdots, n) .
$$

Hence we can deduce as in (6) that

$$
A(x) \sim c x^{n}
$$

Replacing $y$ by $\frac{x^{n}}{c_{1}^{n}}$ in (30), we see

$$
U\left(\frac{x^{n}}{c_{1}^{n}}\right) \leqq E(x)
$$

From (31), (33) and (34), we have

$$
c A(x)<E(x)
$$

for sufficiently large $x$.

Hence we have

THEOREM 2. If we define $A(x)$ and $E(x)$ as in (32), then

$$
c A(x)<E(x)
$$

for sufficiently large $x$.

\section{§ 3. On a density theorem in an algebraic number field.}

For the sets $\mathfrak{A}, \mathfrak{B}, \ldots$ which consist of elements in $I$, we define

$$
\begin{aligned}
\mathfrak{U}+\mathfrak{B} & =\{\gamma: \gamma=\alpha, \beta \text { or } \alpha+\beta ; \alpha \in \mathfrak{A}, \beta \in \mathfrak{B}\}, \\
-\mathfrak{B} & =\{-\boldsymbol{\beta}: \boldsymbol{\beta} \in \mathfrak{B}\}, \\
\mathfrak{A}-\mathfrak{B} & =\mathfrak{A}+(-\mathfrak{B}) .
\end{aligned}
$$

Now we take

$$
\begin{aligned}
& \mathfrak{A}=I, \\
& \mathfrak{S}=\{\zeta: \zeta=\lambda+\mu ; \lambda, \mu \text { primes }\},
\end{aligned}
$$

and write

$$
\begin{aligned}
& \mathfrak{X}[x]=\{\zeta: \zeta \in \mathfrak{A},\|\zeta\| \leqq x\}, \\
& \mathfrak{F}[x]=\left\{\zeta: \zeta \in \mathfrak{F},\|\zeta\| \leqq x ;\|\lambda\|\|\mu\| \leqq c_{1} x\right\}
\end{aligned}
$$


Then the number of elements of $\mathfrak{A}[x]$ and $\mathfrak{E}[x]$ are $A[x]$ and $E[x]$ in (32). Moreover, we define

$$
\mathfrak{B}[x]=\left\{\zeta: \zeta=x_{1} \omega_{1}+x_{2} \omega_{2}+\cdots+x_{n} \omega_{n},-c_{7} x \leqq x_{j} \leqq c_{7} x, x_{j} \in \Gamma\right\}
$$

such that

$$
\mathfrak{A}[x] \subset \mathfrak{B}[x]
$$

by taking $c_{7}$ sufficiently large. Since the number of elements of $\mathfrak{B}[x]$, say $B[x]$, is of order $c x^{n}$, we obtain, for sufficiently large $x$

$$
c B(x)<E(x)
$$

by Theorem 2 and (33) whereas

$$
\mathfrak{S}[x] \subset \mathfrak{B}[x]
$$

by (35).

From (36) and (37), we know that more than $c x^{k^{\prime}}$ elements in the form

$$
\begin{aligned}
& x_{1} \omega_{1}+x_{2} \omega_{2}+\cdots+x_{k} \omega_{k}+x_{k+1} \omega_{k+1}+\cdots+x_{n} \omega_{n}, \\
& -c_{7} x \leqq x_{j} \leqq c_{7} x, \quad x_{j} \in \Gamma \quad(j=1,2, \cdots, k),
\end{aligned}
$$

for suitably fixed $x_{k+1}, x_{k+2}, \cdots, x_{n}$ are contained in $\mathfrak{S}[x]$ for every $k$ $(k=1,2, \cdots, n)$. Hence, $\mathfrak{F}-\mathfrak{F}$ contains more than $c x^{k}$ elements in the form

$$
\begin{aligned}
& y_{k 1} \omega_{1}+y_{k 2} \omega_{2}+\cdots+y_{k k} \omega_{k}, \\
& y_{k j} \in \Gamma,-2 c_{7} x \leqq y_{k j} \leqq 2 c_{7} x
\end{aligned}
$$

for every $k$. Consequently, if we write

$$
\begin{aligned}
& \mathfrak{\Im}_{k}[x]=\left\{y_{k k}: y_{k 1} \omega_{1}+y_{k 2} \omega_{2}+\cdots+y_{k k} \omega_{k} \in \mathfrak{F}-\mathfrak{F},\left|y_{k k}\right| \leqq x\right\}, \\
& C_{k}(x)=\text { the number of elements of } \mathfrak{F}_{k}[x],
\end{aligned}
$$

then

$$
c x<C_{k}(x)
$$

for every $k$, if $x$ is sufficiently large. 
Since $\sqrt{ }-\mathbb{F}$ is contained in $J, J$ contains elements in the form (38). In view of (39), we denote by $a_{k k}$ the smallest positive integer $y_{k k}$ such that

$$
y_{k 1} \omega_{1}+y_{k 2} \omega_{2}+\cdots+y_{k k} \omega_{k} \in J, \quad y_{k j} \in \Gamma,
$$

and write such an element in $J$ which satisfies $y_{k k}=a_{k k}$ as follows.

$$
\zeta_{k}=a_{k 1} \omega_{1}+a_{k 2} \omega_{2}+\cdots+a_{k k} \omega_{k}, \quad a_{k j} \in \Gamma .
$$

Then we can easily deduce that $\zeta_{1}, \zeta_{2}, \cdots, \zeta_{n}$ form a basis of the additive group $J$, and

$$
[I: J]=a_{11} a_{22} \cdots a_{n n}
$$

is finite.

The elements in (38) can be expressed in the form

$$
y_{k 1} \omega_{1}+y_{k 2} \omega_{2}+\cdots+y_{k k} \omega_{k}=z_{k 1} \zeta_{1}+z_{k 2} \zeta_{2}+\cdots+z_{k k} \zeta_{k}
$$

with

$$
-c_{8} x \leqq z_{k j} \leqq c_{8} x, \quad z_{k j} \in \Gamma \text {. }
$$

If we write

$$
\begin{aligned}
& \mathfrak{D}_{k}[x]=\left\{z_{k k}:\left|z_{k k}\right| \leqq x\right\}, \\
& D_{k}(x)=\text { the number of elements of } \mathfrak{D}_{k}[x],
\end{aligned}
$$

then

$$
c x<D_{k}(x)
$$

for every $k$, if $x$ is sufficiently large. Let $\mathfrak{F}$ be the set consisting of all elements in $\mathfrak{F}-\mathfrak{F}$ and $\pm \zeta_{1}, \pm \zeta_{2}, \cdots, \pm \zeta_{n}$. Then $\mathfrak{F}$ is a subset of $J$. Now consider all elements in $\mathfrak{F}$ such form as

$$
f_{k 1} \zeta_{1}+f_{k 2} \zeta_{2}+\cdots+f_{k k} \zeta_{k}, \quad f_{k j} \in \Gamma .
$$

If we write

$$
\begin{aligned}
& \mathfrak{F}_{k}[x]=\left\{f_{k k}:\left|f_{k k}\right| \leqq x\right\} \\
& F_{k}(x)=\text { the number of elements of } \mathfrak{F}_{k}[x]
\end{aligned}
$$


then, by (38),

$$
c x<F_{k}(x)
$$

for every $k$ and $1 \leqq x$. Hence, by the density theorem of Schnirelmann [1], we can deduce that a finite sums of $\mathfrak{F}$ contains such an element as

$$
\xi_{k}=q_{k 1} \zeta_{1}+q_{k 2} \zeta_{2}+\cdots+q_{k k} \zeta_{k}, \quad q_{k j} \in \Gamma
$$

for any given $q_{k k} \in \Gamma$. Hence, every element $\xi$ in $J$ can be expressed in the form

$$
\xi=\xi_{1}+\xi_{2}+\cdots+\xi_{n} \text {. }
$$

Hence we get the desired result.

THEOREM 3. $J$ is of finite index in I and every element in $J$ can be expressed as at most $c$ sums of primes.

\section{Gakushuin University.}

\section{Bibliography}

[1] E. Artin and P. Scherk: On the sum of two sets of integers, Annals of Math., 44 (1943), 138-142.

[2] E. Landau: Über Ideale und Primideale in Idealklassen, Math. Zeitschr., 2 (1918), 52-154.

[3] _ Über einige neuere Fortschritte der additiven Zahlentheorie, Cambridge Tract, 1937.

[4] H. Rademacher: Zur additiven Primzahltheorie algebraischer Zahlkörper, Teile I, II, Abh. Math. Sem. Hamburg 3 (1924) 109-163, 331-378; Teile III, Math. Zeitschr. 27 (1927), 321- 426.

[5] : Über die Anwendung der Viggo Brunschen Methode auf die Theorie der algebraischen Zahlkörper, Berliner Akademie der Wissenschaften, Sitzungsberichte, 1923, 211-218.

[6] C. L. Siegel: Sums of $m$-th powers of algebraic integers, Ann. Math., 46 (1945) 313-339.

[7] I. Vinogradov: Some theorems concerning the theory of primes, Recueil Math., N.S. 2 (1937), 175-195. 